\title{
NARROW DOMAINS AND THE HARNACK INEQUALITY FOR ELLIPTIC EQUATIONS
}

\author{
M. V. SAFONOV
}

Dedicated to Nina N. Ural'tseva

\begin{abstract}
We present a direct proof of Moser's Harnack inequality that does not involve iterations. The method is based on a recursive estimate for solutions in domains of small measure. Such estimates can also be useful for other applications.
\end{abstract}

\section{§1. INTRODUCTION}

We consider second order elliptic operators $L$ in the divergence form

$$
L u:=(D, a D u):=D_{i}\left(a_{i j} D_{j} u\right) .
$$

Here and throughout the paper, the summation convention over repeated indices is enforced, $D$ denotes a symbolic vector with components $D_{i}:=\partial / \partial x_{i}$, and $a:=\left[a_{i j}\right]$ is an $(n \times n)$-matrix of real measurable coefficients satisfying the uniform ellipticity condition

$$
(a \xi, \xi):=a_{i j} \xi_{i} \xi_{j} \geq \nu|\xi|^{2} \quad \forall \xi \in \mathbb{R}^{n}, \text { and } \sum_{i, j}\left|a_{i j}\right|^{2} \leq \nu^{-2} n,
$$

with an ellipticity constant $\nu \in(0,1]$. It is easily seen that $\nu=1$ corresponds to $L=\Delta$.

In the classical theory (see [LU, 1.2] or [GT, 8.1]), the equations $L u=0$ or inequalities $L u \geq 0, L u \leq 0$ in an open set $\Omega \subset \mathbb{R}^{n}$ are understood in an integral sense, and the operators $L$ (which can also include lower order terms) are applied to functions $u$ in the Sobolev space $W^{1,2}(\Omega)$, i.e., $u, D_{i} u \in L^{2}(\Omega)$. The pointwise estimates for such functions, such as the estimates for Hölder constants and the Harnack inequality, hold true under an implicit assumption that the values of $u$ are appropriately modified on a set of zero measure. Fortunately, the most difficult part is to establish such kind of estimates under the additional assumption that all the functions $a_{i j}$ and $u$ are smooth. It is important only that the key estimates depend only on the prescribed constants, such as $n$ and $\nu$, and do not depend on the "additional" smoothness of $a_{i j}$ and $u$. We shall make this point clearer below, in $₫ 6$. For this reason, in order to make the exposition of our method more transparent, we always assume $u \in C(\bar{\Omega})$, and, in the main part of the text, $u \in C^{1}(\bar{\Omega})$ (this is really important in the proof of Theorem 3.4).

In the pioneering work by De Giorgi DG57, as an intermediate step in the proof of the Hölder continuity of solutions of $L u=0$, the following pointwise estimate was derived (in an equivalent formulation).

Theorem 1.1. Let $B_{R}:=B_{R}\left(x_{0}\right)$ be a ball of radius $R>0$ centered at some point $x_{0} \in \mathbb{R}^{n}$. Suppose that $u$ is a function in $W^{1,2}\left(B_{R}\right) \cap C\left(\overline{B_{R}}\right)$ satisfying $u \geq 0$ and

2010 Mathematics Subject Classification. Primary 35J15; Secondary 35B45, 35B65.

Key words and phrases. Second-order elliptic equations, Harnack inequality, measurable coefficients. 
$L u:=(D, a D u) \geq 0$ in $B_{R}$. Then

$$
M:=\sup _{B_{R / 2}} u \leq N \cdot\left(\frac{1}{\left|B_{R}\right|} \int_{B_{R}} u^{2} d x\right)^{1 / 2} \text {, where } N=N(n, \nu) .
$$

The approach in [DG57] takes into consideration the sets

$$
A_{k, r}:=\left\{x \in B_{r}: u(x)>k\right\} \text {, where } k>0 \text { and } r \in(R / 2, R] .
$$

It was shown there that for some sequences $k_{j} \nearrow M$ and $r_{j} \searrow R / 2$, the measures

$$
\left|A_{k_{j}, r_{j}}\right|=\left|B_{r_{j}} \cap\left\{u>k_{j}\right\}\right| \text { tend to } 0 \quad \text { as } j \rightarrow \infty,
$$

which obviously implies $u \leq M$ on $B_{R / 2}$.

Loosely speaking, (1.2) means that the sets $A_{k_{j}, r_{j}}$ become narrow for large $j$.

In the general theory of linear and quasilinear elliptic and parabolic equation, which was created by Ladyzhenskaya and Ural'tseva (see [LU, LSU] and the references therein), the key estimates for solutions are given in terms of the measures $\left|A_{k, r}\right|$. It was pointed out in [LU, II.6] that the authors considered their general technique as a further development of De Giorgi's method.

Estimate (1.1) also appears as a byproduct of the original proof of Moser's Harnack inequality M60, M61, M64]. Namely, in Moser's approach, there exist sequences $p_{j} \nearrow \infty$ and $r_{j} \searrow R / 2$, such that

$$
\|u\|_{L^{p_{j}\left(B_{r_{j}}\right)}} \leq M \text { for all } j
$$

so that the norm of $u$ in $L^{\infty}\left(B_{R / 2}\right)$ does not exceed $M$.

The reader can also find a direct exposition of the methods by De Giorgi and Moser in application to (1.1), the Hölder estimates, and the Harnack inequality in the book by Han and Lin [HL, Chapter 4].

A different kind of iterations appears in the proof of similar results for operators $L$ in the nondivergence form

$$
L u:=(a D, D u):=a_{i j} D_{i} D_{j} u
$$

with measurable $a_{i j}$ satisfying (U). As in (D), for simplicity we do not include lower order terms, and also we do not consider more general parabolic operators. In our joint work with Krylov [KS80, S80] (see also [K85, Chapter 4]), we proved the Hölder estimates and the Harnack inequality in the (ND) case on the grounds of the following facts.

Theorem 1.2. Let an open set $\Omega \subset \mathbb{R}^{n}, x_{0} \in \Omega$, and $R>0$ be such that

$$
\left|\Omega_{R}\right|:=\left|\Omega \cap B_{R}\left(x_{0}\right)\right| \leq \mu \cdot\left|B_{R}\right| \text {, where } \mu=\text { const } \in(0,1) .
$$

(i) Let $u \in W^{2, n}(\Omega) \cap C(\bar{\Omega})$ satisfy

$$
u \geq 0, \quad L u:=(a D u, D u) \geq 0 \text { in } \Omega,
$$

and $u=0$ on $(\partial \Omega) \cap B_{R}\left(x_{0}\right)$. Then

$$
u\left(x_{0}\right) \leq \beta \cdot \sup _{\Omega_{R}} u, \text { where } \beta=\beta(n, \nu, \mu) \in(0,1) .
$$

(ii) (Estimate for narrow domains). Moreover,

$$
\beta(n, \nu, \mu) \rightarrow 0^{+} \text {as } \mu \rightarrow 0^{+} .
$$

Such estimates (also called growth lemmas) have been introduced by Landis La71. for an alternative proof of the results by De Giorgi and Moser mentioned above. In this theorem, part (ii) is proved first: it follows easily from Aleksandrov's estimate in A63. (see also GT, Theorem 9.1]). Then the more difficult part (i) follows by iteration, by 
using a special covering lemma in order to increase the "critical" value of $\mu$ for which (i) holds true.

It was shown in [FS01] that this approach also works in the case of (D), only instead of Aleksandrov's estimate one should use the energy estimate. Note that (1.7) also follows immediately from (1.1). Indeed, by extending $u \equiv 0$ to $B_{R} \backslash \Omega$, we get a function $u$ satisfying all the assumptions of Theorem 1.1. Hence,

$$
u\left(x_{0}\right) \leq M \leq N \cdot\left(\frac{1}{\left|B_{R}\right|} \int_{B_{R}} u^{2} d x\right)^{1 / 2} \leq N \cdot\left(\frac{\left|\Omega_{R}\right|}{\left|B_{R}\right|}\right)^{1 / 2} \cdot \sup _{\Omega_{R}} u,
$$

and (1.4) implies (1.7) with $\beta:=N \mu^{1 / 2}$. This argument is especially simple in the case where $L=\Delta$, the Laplace operator. Then the extended function is subharmonic in $B_{R}$, by the mean value theorem we have

$$
u\left(x_{0}\right) \leq \frac{1}{\left|B_{R}\right|} \int_{\Omega_{R}} u d x \leq \frac{\left|\Omega_{R}\right|}{\left|B_{R}\right|} \cdot \sup _{\Omega_{R}} u
$$

and (1.7) follows with $\beta=\mu$.

Note that though $\beta=\mu$ looks "better" than $\beta=N \mu^{1 / 2}$ for small $\mu$, both these expressions are far from being optimal. In $\S 2$, we use an elementary "scaling" argument to show that from the strict inequality $\beta\left(n, \nu, \mu_{0}\right)<1$ for some $\mu_{0} \in(0,1)$ it follows that in fact convergence in (1.7) is much faster:

$$
\beta \leq \exp \left(-c_{0} \mu^{-1 / n}\right) \text { with } c_{0}=\text { const }>0 .
$$

In its turn, (1.9) follows from a simple recurrent relation $\beta\left(n, \nu, 2^{-n} \mu\right) \leq \beta^{2}(n, \nu, \mu)$. This relation is the core of our approach, it allows us to prove the Harnack inequality and other related facts without using iterations. It only requires a mild technical assumption $u \in C^{1}$. In 93 , we prove a version of estimate (1.7) (Theorem 3.4) and show that it is equivalent to (1.1) (Theorem 3.5). The proof of this equivalence follows the lines of [FS01, Theorem 3.4], we only put aside the crucial part in Lemma 3.6, which is needed again in the proof of the Harnack inequality. Theorem 4.2 in $\$ 4$ can be treated as a version of Theorem 1.2 after replacing $u$ by $(1-u)^{+}$and taking $\beta:=1-\beta_{1}$. In \$5 we prove the Harnack inequality along with its standard applications. The proof is short, because all the essential elements are scattered among the previous sections. Finally, in $\sqrt[6]{6}$ we show how to get rid of an a priori assumption $u \in C^{1}$ and extend the main results to $u \in W^{1,2}$.

Notation. The notation $A:=B$, or $B=: A$, means " $A=B$ by definition".

$x=\left(x_{1}, \ldots, x_{n}\right)$ are vectors or points in $\mathbb{R}^{n},(x, y):=x_{i} y_{i}$ is the scalar product of $x, y \in \mathbb{R}^{n}$ (the summation convention is implied), and $|x|:=(x, x)^{1 / 2}$ is the length of $x \in \mathbb{R}^{n}$.

$B_{R}(x):=\left\{y \in \mathbb{R}^{n}:|y-x|<R\right\}$ is a ball of radius $R>0$ centered at $x \in \mathbb{R}^{n}$.

$\Omega$ is an open set in $\mathbb{R}^{n}$ with boundary $\partial \Omega$ and Lebesgue measure $|\Omega|$. For fixed $x_{0} \in \mathbb{R}^{n}$ and $R>0, \Omega_{R}:=\Omega_{R}\left(x_{0}\right):=\Omega \cap B_{R}\left(x_{0}\right)$.

$\Omega^{\delta}:=\{x \in \Omega: \operatorname{dist}(x, \partial \Omega)>\delta\}$.

$a^{+}:=\max \{a, 0\} . N$ (with or without indices) is a generic notation for various constants depending only on the given quantities, such as $n, \nu$, etc. This dependence is indicated in the parentheses, e.g., $N=N(n, \nu, \ldots)$.

\section{§2. Preliminary Results}

We exploit the fact that the constant $\beta$ in (1.6) depends only on $n, \nu, \mu$, and does not depend on the other characteristics of $\Omega$, the $a_{i j}$, and $u$, so that we can "optimize" the choice in a certain way. 
Lemma 2.1. For fixed $n$ and $\nu$, the constants $\beta(\mu)=\beta(n, \nu, \mu)$ in Theorem 1.2 satisfy

$$
\beta\left(2^{-n} \mu\right) \leq \beta^{2}(\mu) \text { for } \mu \in(0,1) \text {. }
$$

Proof. Let $u$ satisfy (1.6), and suppose that (1.4) holds true with $2^{-n} \mu$ in place of $\mu$, i.e.,

$$
x_{0} \in \Omega \text {, and }\left|\Omega_{R}\right| \leq 2^{-n} \mu \cdot\left|B_{R}\right| .
$$

Since $2^{-n} \cdot\left|B_{R}\right|=\left|B_{R / 2}\right|$, we can use (1.6) with $R / 2$ in place of $R$ :

$$
u\left(x_{0}\right) \leq \beta(\mu) \cdot \sup _{\Omega_{R / 2}\left(x_{0}\right)} u .
$$

By the maximum principle, the supremum on the right-hand side is attained at some point $y_{0} \in \Omega \cap \partial B_{R / 2}\left(x_{0}\right)$, which also satisfies $B_{R / 2}\left(y_{0}\right) \subset B_{R}\left(x_{0}\right)$, whence by (2.2)

$$
\left|\Omega_{R / 2}\left(y_{0}\right)\right| \leq\left|\Omega_{R}\left(x_{0}\right)\right| \leq 2^{-n} \mu \cdot\left|B_{R}\right|=\mu \cdot\left|B_{R / 2}\right| .
$$

Using (2.3) and once again (1.6) with $y_{0}, R / 2$ in place of $x_{0}, R$ (respectively), we get

$$
u\left(x_{0}\right) \leq \beta(\mu) \cdot u\left(y_{0}\right) \leq \beta^{2}(\mu) \cdot \sup _{\Omega_{R / 2}\left(y_{0}\right)} u \leq \beta^{2}(\mu) \cdot \sup _{\Omega_{R}\left(x_{0}\right)} u .
$$

The lemma is proved.

By itself, estimate (2.1) does not guarantee the convergence $\beta(\mu) \rightarrow 0^{+}$as $\mu \rightarrow 0^{+}$, because it holds true for $\beta \equiv 1$. We really need $\beta\left(\mu_{0}\right)<1$ for some $\mu_{0} \in(0,1)$.

Corollary 2.2. Let $\beta(\mu)$ be a positive monotone nondecreasing function on $(0,1)$ such that $\beta\left(\mu_{0}\right) \in(0,1)$ for some $\mu_{0} \in(0,1)$. Then from (2.1) it follows that

$$
\beta(\mu) \leq \beta_{0}(\mu):=\exp \left(-c_{0} \cdot \mu^{-1 / n}\right) \quad \text { on }\left(0, \mu_{0}\right],
$$

where

$$
c_{0}:=-\frac{1}{2} \cdot \mu_{0}^{1 / n} \ln \beta\left(\mu_{0}\right)>0 .
$$

Proof. Obviously,

$$
\left(0, \mu_{0}\right]=\bigcup_{j=0}^{\infty} \Delta_{j}, \text { where } \Delta_{j}:=\left[2^{-n(j+1)} \mu_{0}, 2^{-n j} \mu_{0}\right] .
$$

We have

$$
\beta_{0}\left(2^{-n} \mu\right)=\exp \left(-2 c_{0} \mu^{-1 / n}\right)=\beta_{0}^{2}(\mu) \text { on }\left(0, \mu_{0}\right],
$$

and by the choice of $c_{0}$ in (2.5),

$$
\beta_{0}\left(2^{-n} \mu_{0}\right)=\exp \left(-2 c_{0} \mu_{0}^{-1 / n}\right)=\exp \left(\ln \left(\beta\left(\mu_{0}\right)\right)\right)=\beta\left(\mu_{0}\right) .
$$

Since both functions $\beta(\mu)$ and $\beta_{0}(\mu)$ are monotone nondecreasing, it follows that

$$
\beta(\mu) \leq \beta\left(\mu_{0}\right)=\beta_{0}\left(2^{-n} \mu_{0}\right) \leq \beta_{0}(\mu) \text { on } \Delta_{0}:=\left[2^{-n} \mu_{0}, \mu_{0}\right] .
$$

Suppose that $\beta(\mu) \leq \beta_{0}(\mu)$ on $\Delta_{j}$ for some integer $j \geq 0$. For such $\mu$, we have

$$
\beta\left(2^{-n} \mu\right) \leq \beta^{2}(\mu) \leq \beta_{0}^{2}(\mu)=\beta_{0}\left(2^{-n} \mu\right) .
$$

Since each point in $\Delta_{j+1}$ can be represented as $2^{-n} \mu$ with $\mu \in \Delta_{j}$, we have $\beta \leq \beta_{0}$ on $\Delta_{j+1}$. By induction, $\beta \leq \beta_{0}$ on $\Delta_{j}$ for each integer $j \geq 0$, which completes the proof of (2.4). 


\section{§3. Estimates FOR SUbSOlutions}

Throughout this section, we fix an open set $\Omega \subset \mathbb{R}^{n}$, a point $x_{0} \in \Omega$, a constant $R>0$, and denote $B_{R}:=B_{R}\left(x_{0}\right), \Omega_{R}:=\Omega \cap B_{R}$. Here we derive local estimates for functions $u$ satisfying the inequality $L u:=(D, a D u) \geq 0$ in $\Omega \subset \mathbb{R}^{n}$ in a weak sense, i.e., by formal integration by parts,

$$
\int_{\Omega}(a D u, D \eta) d x \leq 0 \quad \forall \eta \in C_{0}^{\infty}(\Omega), \quad \eta \geq 0 .
$$

By approximation, this property is easily extended to functions $\eta \in C^{1}(\bar{\Omega})$ satisfying $u=0$ on $\partial \Omega$. In the classical theory (see [LU, III.4], GT, Chapter 8]), these relations are considered for $u \in W^{1,2}(\Omega)$, so that $D u \in L^{2}(\Omega)$. For the simplicity of presentation, especially for the proof of Theorem 3.4 we impose the technical restriction $u \in C^{1}(\bar{\Omega})$. The extension of main results to $u \in W^{1,2}(\Omega)$ is pretty standard. For completeness and the reader's convenience, we provide more details at the end, in $₫ 6$.

We start with an energy estimate, which is well known even for more general parabolic equations (see estimate (III.2.18) in [LSU]). It served as an initial step in Moser's iterations (see [M61, Lemma 4], and also estimate (IX.5.7) in [LU]).

Lemma 3.1 (Energy estimate). Let $u$ be a functions in $C^{1}(\bar{\Omega})$ satisfying

$$
u \geq 0, \quad L u:=(D, a D u) \geq 0 \quad \text { in } \Omega ; \quad \text { and } u=0 \quad \text { on }(\partial \Omega) \cap B_{R} .
$$

Take a function $\zeta \in C^{1}\left(\overline{B_{R}}\right)$ satisfying

$$
0 \leq \zeta \leq 1, \quad|D \zeta| \leq \frac{4}{R} \quad \text { in } \quad B_{R} ; \quad \zeta \equiv 1 \quad \text { on } \quad B_{R / 2}, \quad \zeta=0 \quad \text { on } \quad \partial B_{R} .
$$

Then

$$
\int_{\Omega_{R}}|D(\zeta u)|^{2} d x \leq \frac{N}{R^{2}} \int_{\Omega_{R}} u^{2} d x, \text { where } N=N(n, \nu) .
$$

Proof. The function $\eta \in C^{1}(\bar{\Omega})$ satisfies

$$
D \eta=\zeta^{2} D u+2 \zeta u D \zeta \text { in } \Omega_{R}, \text { and } \eta=0 \text { on } \partial \Omega_{R} .
$$

From (3.1) it follows that

$$
\nu \int_{\Omega_{R}} \zeta^{2}|D u|^{2} d x \leq \int_{\Omega_{R}} \zeta^{2}(a D u, D u) d x \leq-2 \int_{\Omega_{R}} \zeta u(a D u, D \zeta) d x .
$$

By the Cauchy-Schwarz inequality and the estimate for $|D \zeta|$ in (3.3), the last expression does not exceed

$$
\frac{\nu}{2} \int_{\Omega_{R}} \zeta^{2}|D u|^{2} d x+\frac{N}{R^{2}} \int_{\Omega_{R}} u^{2} d x, \text { where } N=N(n, \nu)>0 .
$$

This yields the esimate

$$
\int_{\Omega_{R}} \zeta^{2}|D u|^{2} d x \leq \frac{N}{R^{2}} \int_{\Omega_{R}} u^{2} d x .
$$

Now the desired estimate (3.4) follows from

$$
|D(\zeta u)|^{2}=|\zeta D u+u D \zeta|^{2} \leq 2 \zeta^{2}|D u|^{2}+2 u^{2}|D \zeta|^{2} .
$$

The lemma is proved.

The following lemma is a varsion of estimate (II.2.12) in [LU].

Lemma 3.2. Let $u$ be a function in $C^{1}(\bar{\Omega})$ satisfying $u=0$ on $\partial \Omega$. Then

$$
\|u\|_{1, \Omega}:=\int_{\Omega}|u| d x \leq|\Omega|^{\frac{1}{2}+\frac{1}{n}}\|D u\|_{2, \Omega} .
$$


Proof. The Gagliardo-Nirenberg-Sobolev inequality (see the proof of Theorem 1 in $\mathrm{E}$, $5.6]$ for $p=1$ ) can be written as

$$
\|u\|_{q, \Omega}:=\left(\int_{\Omega}|u|^{q} d x\right)^{1 / q} \leq\|D u\|_{1, \Omega}, \text { where } q:=\frac{n}{n-1} .
$$

In the case where $|\Omega|=1$, by the Hölder inequality, the norm $\|u\|_{p, \Omega}$ is a monotone nondecreasing function of $p$, and (3.5) follows immediately from (3.6).

In the general case, take $\lambda:=|\Omega|^{1 / n}$ and substitute $x=\lambda y, d x=\lambda^{n} d y$. Then the function

$$
v(y):=u(\lambda y) \text { in } \Omega_{0}:=\left\{y \in \mathbb{R}^{n}: \lambda y \in \Omega\right\}
$$

satisfies the above assumptions with $\left|\Omega_{0}\right|=\lambda^{-n} \cdot|\Omega|=1$. Therefore,

$$
\|u\|_{1, \Omega}=\lambda^{n} \cdot\|v\|_{1, \Omega_{0}} \leq \lambda^{n} \cdot\|D v\|_{2, \Omega_{0}}=\lambda^{n+1} \cdot\|D u(\lambda y)\|_{2, \Omega_{0}}=\lambda^{\frac{n}{2}+1} \cdot\|D u\|_{2, \Omega} .
$$

By the choice of $\lambda$, the last expression coincides with the right-hand side in (3.5).

Combining Lemmas 3.1 and 3.2, we derive the following useful estimate.

Lemma 3.3. Let u be a function in $C^{1}(\bar{\Omega})$ satisfying the assumptions (3.2) in Lemma 3.1 . Suppose that for some positive constants $\mu, \beta$, and $c$, we have inequality (1.4) and

$$
\left|\Omega_{R / 2} \cap\{u>\beta M\}\right| \geq c \mu \cdot\left|B_{R}\right|, \text { where } M:=\sup _{\Omega_{R}} u .
$$

Then

$$
c \beta \leq N_{0} \mu^{\frac{1}{n}}, \text { where } N_{0}=N_{0}(n, \nu)>0 .
$$

Proof. From properties (1.4) and (3.7) it follows that

$$
c \beta M \cdot\left|\Omega_{R}\right| \leq c \mu \beta M \cdot\left|B_{R}\right| \leq \int_{\Omega_{R / 2}} u d x \leq \int_{\Omega_{R}} \zeta u d x,
$$

where $\zeta$ is a function in (3.3). Applying Lemma 3.2 to the function $\zeta u \in C^{1}\left(\overline{\Omega_{R}}\right)$, and then using the energy estimate (3.4), we obtain

$$
\begin{aligned}
\int_{\Omega_{R}} \zeta u d x & =:\|\zeta u\|_{1, \Omega_{R}} \leq\left|\Omega_{R}\right|^{\frac{1}{2}+\frac{1}{n}} \cdot\|D(\zeta u)\|_{2, \Omega_{R}} \\
& \leq \frac{N}{R} \cdot\left|\Omega_{R}\right|^{\frac{1}{2}+\frac{1}{n}} \cdot\|u\|_{2, \Omega_{R}} \leq \frac{N M}{R} \cdot\left|\Omega_{R}\right|^{1+\frac{1}{n}} .
\end{aligned}
$$

Comparing with (3.9) and again using (1.4), we see that

$$
c \beta \leq \frac{N}{R} \cdot\left|\Omega_{R}\right|^{\frac{1}{n}} \leq \frac{N}{R} \cdot \mu^{\frac{1}{n}} \cdot\left|B_{R}\right|^{\frac{1}{n}}=: N_{0} \mu^{\frac{1}{n}} .
$$

Thus, the lemma is proved.

The next theorem includes property (1.7) in Theorem 1.2 (ii) for small $\mu>0$. Note that the rate of convergence $\beta \rightarrow 0$ as $\mu \rightarrow 0^{+}$can be improved, in accordance with Lemma 2.1 and Corollary 2.2 .

Theorem 3.4. Suppose that the measure of $\Omega_{R}$ satisfies (1.4) with a small constant $\mu \in(0,1)$, namely,

$$
\beta:=4^{n+1} N_{0} \mu^{\frac{1}{n}} \leq \frac{1}{2},
$$

where $N_{0}=N_{0}(n, \nu)$ is a constant in (3.8). Then for any function $u \in C^{1}(\bar{\Omega})$ satisfying (3.2), we have the estimate

$$
u\left(x_{0}\right) \leq \beta \cdot \sup _{\Omega_{R}} u
$$


Proof. Fix $\varepsilon>0$. We show that for each ball $B_{r}(y) \subset B_{R}:=B_{R}\left(x_{0}\right)$ and $k \geq 0$, from

$$
\left|\Omega_{r}(y) \cap\{u>k\}\right| \leq \mu \cdot\left|B_{r}\right|
$$

it follows that

$$
(u-k)^{+}(y) \leq \varepsilon \sqrt{r}+\beta \cdot M, \text { where } M:=\sup _{\Omega_{r}(y)}(u-k)^{+} .
$$

Since the constant $\beta$ does not depend on $\varepsilon$, the estimate remains valid for $\varepsilon=0$, and the desired estimate (3.11) follows by taking $k=0, y=x_{0}$, and $r=R$.

Our assumption $u \in C^{1}(\bar{\Omega})$ together with (3.12) guarantees that

$$
(u-k)^{+}(y) \leq r \cdot \sup |D u| \leq \varepsilon \sqrt{r}
$$

for small $r>0$, so that (3.13) is trivial. Therefore, it suffices to prove that (3.12) implies (3.13) assuming that we already have a similar property with $r / 4$ in place of $r$, for arbitrary $k, y$, and $r$ under consideration.

Based on (3.12), we can apply Lemma 3.3 to the function $u-k$ on the open set $\Omega_{r}(y) \cap\{u>k\}$, which satisfies (1.1) with $x_{0}=y, R=r$. By our choice of $\beta$ in (3.10), we have

$$
4^{-n} \cdot \frac{\beta}{2}>N_{0} \mu^{\frac{1}{n}}
$$

i.e., inequality (3.8) fails with $c:=4^{-n}$ and $\beta / 2$ in place of $\beta$. Therefore, (3.7) must fail as well:

$$
\left|\Omega_{r / 2}(y) \cap\left\{u-k>\frac{\beta M}{2}\right\}\right|<4^{-n} \mu \cdot\left|B_{r}\right|=\mu \cdot\left|B_{r / 4}\right| .
$$

Set $k_{0}:=k+\beta M / 2$. Inequality (3.14) implies (3.12) with $k_{0}, r / 4$ in place of $k, r$, respectively. Since we assume that (3.13) is valid for $r / 4$, we get

$$
\left(u-k_{0}\right)^{+}(y) \leq \frac{\varepsilon \sqrt{r}}{2}+\beta \cdot \sup _{\Omega_{r / 4}(y)}\left(u-k_{0}\right)^{+} .
$$

Further, note that for each point $z \in \Omega_{r / 4}(y)$, we have $\Omega_{r / 4}(z) \subset \Omega_{r / 2}(y)$, so by the same argument, we get a rough estimate

$$
\left(u-k_{0}\right)^{+}(z) \leq \frac{\varepsilon \sqrt{r}}{2}+\beta \cdot \sup _{\Omega_{r / 4}(z)}\left(u-k_{0}\right)^{+}<\frac{\varepsilon \sqrt{r}}{2}+\beta \cdot M .
$$

Combining these estimates, we arrive at

$$
\left(u-k_{0}\right)^{+}(y)<\varepsilon \sqrt{r}+\beta^{2} M .
$$

Finally, since $k_{0}-k=\beta M / 2$ and $\beta \leq 1 / 2$, we obtain estimate (3.13):

$$
(u-k)^{+}(y) \leq\left(u-k_{0}\right)^{+}(y)+\frac{\beta M}{2} \leq \varepsilon \sqrt{r}+\beta\left(\beta+\frac{1}{2}\right) M \leq \varepsilon \sqrt{r}+\beta M .
$$

As has already been pointed out, the desired estimate (3.11) follows.

Theorem 3.4 automatically yields a pointwise estimate in terms of $L^{p}$ norms. This is an "elliptic" varsion of Theorem 3.4 in FS01 for the parabolic case. Note that one can apply this theorem with $p=2$ and an arbitrary $y \in \Omega_{R / 2}:=\Omega_{R / 2}\left(x_{0}\right)$ in place of $x_{0}$ :

$$
u^{2}(y) \leq \frac{N}{\left|B_{R}\right|} \int_{\Omega_{R / 2}(y)} u^{2} d x .
$$

Since $\Omega_{R / 2}(y) \subset \Omega_{R}$, estimate (1.1) in Theorem 1.1 follows. 
Theorem 3.5. Let $u \in C^{1}(\bar{\Omega})$ satisfy (3.2). Then for arbitrary $p>0$ we have

$$
u^{p}\left(x_{0}\right) \leq \frac{N}{\left|B_{R}\right|} \int_{\Omega_{R}} u^{p} d x, \text { where } N=N(n, \nu, p)>0 .
$$

If $B_{R} \subset \Omega$, then $\Omega_{R}=B_{R}$ and there is no boundary condition in (3.2).

The proof of this theorem follows easily from the next claim.

Lemma 3.6. Under the assumptions of the previous theorem, for arbitrary $\gamma>0$, there is a constant $\mu_{0}=\mu_{0}(n, \nu, \gamma) \in(0,1)$ and a ball $B_{\rho}(y) \subset B_{2 \rho}(y) \subset B_{R}$ such that

$$
\left|\Omega_{\rho}(y) \cap\{u>k\}\right|>\mu_{0} \cdot\left|B_{\rho}\right|, \text { where } k:=2^{-\gamma-1}\left(\frac{R}{\rho}\right)^{\gamma} u\left(x_{0}\right) .
$$

Proof of Theorem 3.5. Take $\gamma:=n / p$. Then by (3.16) we have

$$
\int_{\Omega_{\rho}(y)} u^{p} d x \geq \mu_{0} \cdot\left|B_{\rho}\right| \cdot k^{p}=2^{-n-p} \mu_{0} \cdot\left|B_{R}\right| \cdot u^{p}\left(x_{0}\right),
$$

and (3.15) follows with $N:=2^{n+p} / \mu_{0}$.

Proof of Lemma 3.6. Denote $d(x):=R-\left|x-x_{0}\right|=\operatorname{dist}\left(x, \partial B_{R}\right)$ on $\Omega_{R}$. Then $d\left(x_{0}\right)=R$, and

$$
R^{\gamma} u\left(x_{0}\right) \leq M:=\sup _{\Omega_{R}} d^{\gamma} u
$$

Since $d^{\gamma} u=0$ on $\partial \Omega_{R}$, the supremum here is attained at some point $y \in \Omega_{R}$. Set $\rho:=d(y) / 2$, so that $M=(2 \rho)^{\gamma} u(y)$. Obviously, $d \geq \rho$ on $\Omega_{\rho}(y)$, whence

$$
\sup _{\Omega_{\rho}(y)} u \leq \rho^{-\gamma} \sup _{\Omega_{\rho}(y)} d^{\gamma} u=\rho^{-\gamma}\left(d^{\gamma} u\right)(y)=2^{\gamma} u(y)
$$

and the function $v(x):=u(x)-u(y) / 2$ satisfies

$$
v(y)=2^{-1} u(y) \geq 2^{-\gamma-1} \sup _{\Omega_{\rho}(y)} u>2^{-\gamma-1} \sup _{\Omega_{\rho}(y)} v .
$$

Note that by (3.17),

$$
k:=2^{-\gamma-1}\left(\frac{R}{\rho}\right)^{\gamma} u\left(x_{0}\right) \leq 2^{-\gamma-1} \rho^{-\gamma} M=\frac{u(y)}{2} .
$$

Now we take $\mu_{0}=\mu_{0}(n, \nu, \gamma)$ from the identity

$$
2^{-\gamma-1}=4^{n+1} N_{0} \mu_{0}^{\frac{1}{n}},
$$

where $N_{0}=N_{0}(n, \nu)$ is a constant in (3.10) in Theorem 3.4, so that 3.10 holds true with $\beta:=2^{-\gamma-1}, \mu:=\mu_{0}$. On the other hand, inequality (3.18) means that (3.11) fails for the function $v(x)$ in $\Omega_{\rho}(y) \cap\{v>0\}$. Therefore, (1.1) fails as well, i.e.,

$$
\left|\Omega_{\rho}(y) \cap\{v>0\}\right|>\mu_{0} \cdot\left|B_{\rho}\right| .
$$

By (3.19), we have $\{v>0\} \subset\{u>k\}$, and estimate (3.16) follows. 


\section{$\S 4$. Estimates For Supersolutions}

We need the following estimate.

Lemma 4.1. Suppose that an open set $\Omega$ and a ball $B_{1}$ of radius 1 in $\mathbb{R}^{n}$ satisfy

$$
\left|\Omega_{1}\right|:=\left|\Omega \cap B_{1}\right| \leq \mu \cdot\left|B_{1}\right| \text {, where } \mu \in(0,1) .
$$

Then for any function $v \in C^{1}(\bar{\Omega})$ such that $v=0$ on $(\partial \Omega) \cap B_{1}$, we have

$$
\int_{\Omega_{1}} v^{2} d x \leq N \cdot \int_{\Omega_{1}}|D v|^{2} d x, \text { where } N=N(n, \mu)>0 .
$$

This lemma follows immediately from [M60, Lemma 2] with $N:=B_{1} \backslash \Omega$, (see also [LU, Lemma II.3.8]).

One can also prove (4.1) by a contradiction argument, which does not provide an explicit expression for the constant $N(n, \mu)$ (see [HL, Lemma 4.8]). The following lemma is also a version of [HL, Theorem 4.9], see also the proof of (IX.5.15) in [LU].

Theorem 4.2. Let $u$ be a function in $C^{1}\left(\overline{B_{2 R}}\right)$ satisfying

$$
u \geq 0 \text { and } L u:=(D, a D u) \leq 0 \text { in } B_{2 R}
$$

in a weak sense, i.e.,

$$
\int_{B_{2 R}}\left(a D u, D \eta_{0}\right) d x \geq 0 \quad \forall \eta_{0} \in C_{0}^{\infty}\left(B_{2 R}\right), \quad \eta_{0} \geq 0 .
$$

Suppose that

$$
\left|B_{R} \cap\{u<1\}\right| \leq \mu \cdot\left|B_{R}\right|, \text { where } \mu \in(0,1)
$$

Then

$$
u \geq \beta_{1}=\beta_{1}(n, \nu, \mu)>0 \text { on } B_{R / 2} .
$$

Proof. Step 1. By rescaling $x \rightarrow R^{-1} x$, the proof reduces to the case where $R=1$. In addition, without loss of generality, we may assume that $u \geq \delta=$ const $>0$ on $B_{2}$, so that

$$
v:=G(u):=-\ln u \in C^{1}\left(\overline{B_{2}}\right) .
$$

Note that since $G^{\prime}(u)=-u^{-1}<0$ and $G^{\prime \prime}(u)=u^{-2}>0$, we have $L v:=(D, a D v) \geq 0$ in $B_{2}$. Indeed, for an arbitrary $0 \leq \eta \in C^{1}\left(\overline{B_{2}}\right), \eta=0$ on $\partial B_{2}$ we also have $0 \leq \eta_{0}:=$ $-G^{\prime}(u) \eta \in C^{1}\left(\overline{B_{2}}\right), \eta_{0}=0$ on $\partial B_{2}$, and

$$
D \eta_{0}=-G^{\prime}(u) D \eta-G^{\prime \prime}(u) \eta D u, \quad D v=G^{\prime}(u) D u .
$$

Therefore,

$$
\begin{aligned}
\int_{B_{2}}(a D v, D \eta) d x & =\int_{B_{2}}\left(a D u, G^{\prime}(u) D \eta\right) d x \\
& =-\int_{B_{2}}\left(a D u, D \eta_{0}\right) d x-\int_{B_{2}} G^{\prime \prime}(u) \eta \cdot(a D u, D u) d x \leq 0,
\end{aligned}
$$

because of (4.2) and the ellipticity condition (U). By definition, this means that $L v \geq 0$ in $B_{2}$ in a weak sense. Moreover, since

$$
G^{\prime \prime}(u)(a D u, D u)=(a D v, D v) \geq \nu|D v|^{2},
$$

we can write a stronger inequality

$$
\int_{B_{2}}(a D v, D \eta) d x \leq-\nu \int_{B_{2}} \eta|D v|^{2} d x \quad \forall \eta \in C_{0}^{\infty}\left(B_{2}\right), \quad \eta \geq 0 .
$$


Take $\eta:=\zeta^{2}$, where $\zeta$ is a standard cut-off function in (3.3) for the ball $B_{2}$, i.e.,

$$
0 \leq \zeta \leq 1, \quad|D \zeta| \leq 2 \text { in } B_{2} ; \quad \zeta \equiv 1 \text { on } B_{1}
$$

Then from (4.5) it follows that

$$
\nu \int_{B_{2}} \zeta^{2}|D v|^{2} d x \leq-2 \int_{B_{2}} \zeta \cdot(a D v, D \zeta) d x \leq N \int_{B_{2}} \zeta|D v| d x
$$

with $N=N(n, \nu)>0$. By the Cauchy-Schwarz inequality, the last expression does not exceed

Therefore,

$$
\frac{\nu}{2} \int_{B_{2}} \zeta^{2}|D v|^{2} d x+N
$$

$$
\int_{B_{1}}|D v|^{2} d x \leq \int_{B_{2}} \zeta^{2}|D v|^{2} d x \leq N=N(n, \nu) .
$$

Step 2. Setting $\Omega:=B_{2} \cap\{u<1\}=B_{2} \cap\{v>0\}$, we observe that

$$
v \geq 0, \quad L v \geq 0 \text { in } \Omega_{1}:=\Omega \cap B_{1} ; \text { and } v=0 \text { on }(\partial \Omega) \cap B_{1} .
$$

Moreover, by (4.3), we also have $\left|\Omega_{1}\right| \leq \mu \cdot\left|B_{1}\right|$. Then we can use Lemma 4.1, which in combination with (4.6) provides the estimate

$$
\int_{\Omega_{1}} v^{2} d x \leq N \cdot \int_{\Omega_{1}}|D v|^{2} d x \leq N=N(n, \nu, \mu) .
$$

Step 3. Finally, for each point $y \in \Omega_{1 / 2}$, we can apply Theorem 3.5 to the function $v$ on $\Omega_{1 / 2}(y) \subset \Omega_{1}$ with $p=2$. Then

$$
v^{2}(y) \leq N \int_{\Omega_{1 / 2}(y)} v^{2} d x \leq N \int_{\Omega_{1}} v^{2} d x \leq N=N(n, \nu, \mu) .
$$

Since this estimate is uniform for $y \in \Omega_{1 / 2}:=B_{1 / 2} \cap\{u<1\}$, we have

$$
u=e^{-v} \geq \beta_{1}=\beta_{1}(n, \nu, \mu)>0 \text { on } \Omega_{1 / 2} .
$$

On the complementary set $B_{1 / 2} \backslash \Omega_{1 / 2}$, we have $u \geq 1$, so that the inequality $u \geq \beta_{1}$ actually holds true on the entire ball $B_{1 / 2}$, i.e., we have (4.4).

Corollary 4.3. Suppose that $u \in C^{1}\left(\overline{B_{4 r}}\right)$ satisfies $u \geq 0$ and $L u \leq 0$ in $B_{4 r}$. Then

$$
m(\rho):=\inf _{B_{\rho}} u \leq\left(\frac{2 r}{\rho}\right)^{\gamma} m(r) \text { for } 0<\rho \leq r, \text { where } \gamma=\gamma(n, \nu)>0 .
$$

Proof. Fix $\rho \in(0, r / 2]$ and apply Theorem 4.2 to the function $u_{0}(x):=u(x) / m(\rho)$ with $R=4 \rho \leq 2 r$. We have $u_{0} \geq 1$ on $B_{R / 4}=B_{\rho}$; therefore,

$$
\left|B_{R} \cap\left\{u_{0}<1\right\}\right| \leq\left|B_{R} \backslash B_{R / 4}\right|=\mu \cdot\left|B_{R}\right| \text {, where } \mu:=1-4^{-n} .
$$

By (4.4) we have $u_{0} \geq \beta_{1}>0$ on $B_{R / 2}=B_{2 \rho}$, where $\beta_{1} \in(0,1)$ depends only on $n$ and $\nu$. Then $u \geq \beta_{1} m(\rho)$ on $B_{2 \rho}$, so that $m(2 \rho) \geq \beta_{1} m(\rho)$, and

$$
m(\rho) \leq 2^{\gamma} m(2 \rho) \text {, where } \gamma=\gamma(n, \nu):=-\log _{2} \beta_{1}>0 .
$$

For each $\rho \in(0, r]$, one can write

$$
2^{-j-1} r<\rho \leq 2^{-j} r, \quad 2^{j} \leq \frac{r}{\rho} \text { for some integer } j \geq 0 .
$$

Iterating (4.8) gives the desired estimate (4.7):

$$
m(\rho) \leq m\left(2^{-j-1} r\right) \leq 2^{(j+1) \gamma} m(r) \leq\left(\frac{2 r}{\rho}\right)^{\gamma} m(r) .
$$

The corollary is proved. 


\section{$\S 5$. The Harnack Inequality}

Now we show how Lemma 3.6. Theorem 4.2, and Corollary 4.3 work for the proof of Moser's Harnack inequality [M61. The results in this section are true without a priori assumption $u \in C^{1}$. We discuss this separately in $₫ 6$.

Theorem 5.1 (Harnack Inequality). Let $u \in C^{1}\left(\overline{B_{14 R}}\right)$ satisfy

$$
u \geq 0 \text { and } L u:=(D, a D u)=0 \text { in } B_{14 R} .
$$

Then for a concentric ball $B_{R}$,

$$
\sup _{B_{R}} u \leq N_{1} \cdot \inf _{B_{R}} u, \text { where } N_{1}=N_{1}(n, \nu)>1 .
$$

Proof. Without loss of generality, we assume that $R=1$. Fix an arbitrary point $x_{0} \in B_{1}$. Let $\gamma=\gamma(n, \nu)>0$ be a constant in Corollary 4.3. By Lemma 3.6, there is a constant $\mu_{0}=\mu_{0}(n, \nu) \in(0,1)$ and a ball $B_{\rho}(y) \subset B_{2 \rho}(y) \subset B_{1}\left(x_{0}\right)$ such that

$$
\left|B_{\rho} \cap\{u>k\}\right|>\mu_{0} \cdot\left|B_{\rho}\right|, \text { where } k:=2^{-\gamma-1} \rho^{-\gamma} u\left(x_{0}\right) .
$$

We can rewrite this inequality as

$$
\left|B_{\rho} \cap\left\{k^{-1} u<1\right\}\right|<\mu \cdot\left|B_{\rho}\right|, \text { where } \mu:=1-\mu_{0} \in(0,1),
$$

and apply Theorem 4.2 with $R=\rho$ to the function $k^{-1} u$ on the ball $B_{\rho}(y) \subset B_{2 \rho}(y) \subset$ $B_{1}\left(x_{0}\right)$. This implies

$$
u \geq \beta_{1} k \text { on } B_{\rho / 2}(y), \text { where } \beta_{1}=\beta_{1}(n, \nu)>0 .
$$

Note that $B_{1} \subset B_{3}(y) \subset B_{12}(y) \subset B_{14}$, so that we can use Corollary 4.3 with $r=3$ :

$$
\beta_{1} k \leq \inf _{B_{\rho / 2}(y)} u \leq\left(\frac{12}{\rho}\right)^{\gamma} \inf _{B_{3}(y)} u \leq\left(\frac{12}{\rho}\right)^{\gamma} \inf _{B_{1}} u .
$$

From the expression for $k$ in (5.2), we conclude that

$$
\beta_{1} u\left(x_{0}\right) \leq 2 \cdot 24^{\gamma} \cdot \inf _{B_{1}} u \text {. }
$$

Since $x_{0}$ is an arbitrary point in $B_{1}$, inequality (5.1) follows with $N_{1}=N_{1}(n, \nu):=$ $2 \cdot 24^{\gamma} / \beta_{1}$.

The theorem is proved.

One can reformulate the Harnack inequality in the following equivalent way, which is more convenient for some applications.

Theorem 5.2. Let $\Omega$ be a bounded open set in $\mathbb{R}^{n}$ such that diam $\Omega \leq K \delta$, where $K$ and $\delta$ are positive constants, and let $\Omega^{\delta}:=\Omega \cap\{\operatorname{dist}(x, \partial \Omega)>\delta\}$ be connected. Suppose that $u \in C^{1}(\Omega)$ satisfies $u \geq 0$ and $L u=0$ in $\Omega$. Then

$$
\sup _{\Omega^{\delta}} u \leq N_{2} \cdot \inf _{\Omega^{\delta}} u, \text { where } N_{2}=N_{2}(n, \nu, K)>1 .
$$

Proof. Any two points $x, y \in \Omega^{\delta}$ can be connected in $\Omega^{\delta}$ by a curve $\gamma$ of length $|\gamma| \leq N \delta$, where $N=N(n, K)$. One can choose a finite sequence $x_{0}=x, x_{1}, x_{2}, \ldots, x_{M}=y$ in $\gamma$ such that $\left|x_{j}-x_{j-1}\right|<\delta / 14$ for all $j=1,2, \ldots, M$, where $M$ does not exceed a constant $N_{0}=N_{0}(n, K)$. Iterating (3.2) with $R:=\delta / 14$, we get

$$
u(x)=u\left(x_{0}\right) \leq N_{1} u\left(x_{1}\right) \leq N_{1}^{2} u\left(x_{2}\right) \leq \ldots N_{1}^{M} u\left(x_{M}\right)=N_{1}^{M} u(y),
$$

and (5.3) follows with $N_{2}=N_{2}(n, K):=N_{1}^{N_{0}}$.

Corollary 5.3. In Theorem 5.1, the ball $B_{14 R}$ can be replaced by $B_{c R}$ with $c=$ const $>1$. Then (5.1) holds true with $N_{1}=N_{1}(n, \nu, c)>1$. 
Proof. In Theorem [5.2, take $\Omega:=B_{c R}$ and $\delta:=(1-c) R$. Then

$$
\Omega^{\delta}=B_{R}, \quad \operatorname{diam} \Omega=2 c R+K \delta \text { with } K:=2 c \cdot(1-c)^{-1},
$$

and the statement follows from (5.3).

Another standard consequence of the Harnack inequality is the Hölder continuity of solutions (see [M61, Section 5]).

Theorem 5.4 (Hölder continuity). Let $u \in C^{1}(\Omega)$ satisfy Lu $=0$ in $\Omega$. Then

$$
\omega(\rho):=\sup _{B_{\rho}} u-\inf _{B_{\rho}} u \leq\left(\frac{2 \rho}{r}\right) \omega(r) \text { for } 0<\rho<r<R, \quad B_{R} \subset \Omega .
$$

\section{$\S 6$. Smooth approximation}

Now we show how the results of the preceding sections, which were proved for solutions $u \in C^{1}$, can be extended to the functions $u \in W^{1,2}(\Omega)$ satisfying $L u:=(D, a D u)=0$ in $\Omega$, i.e.,

$$
\int_{\Omega}(a D u, D \eta)=0 \quad \forall \eta \in C_{0}^{\infty}(\Omega)
$$

Consider approximation of $u$ and $a_{i j}$ by standard mollifiers $u^{(\varepsilon)}:=\varphi_{\varepsilon} * u$ and $a_{i j}^{(\varepsilon)}:=$ $\varphi_{\varepsilon} * a_{i j}$, where

$$
0 \leq \varphi \in C_{0}^{\infty}\left(B_{\varepsilon}\right), \quad \int_{B_{\varepsilon}} \varphi_{\varepsilon} d x=1
$$

For $0<\varepsilon \leq \delta$, we have $u^{(\varepsilon)}, a_{i j}^{(\varepsilon)} \in C^{\infty}\left(\overline{\Omega^{\delta}}\right)$,

$$
u^{(\varepsilon)} \rightarrow u \text { in } W^{1,2}\left(\Omega^{\delta}\right), \quad a_{i j}^{(\varepsilon)} \rightarrow a_{i j} \text { a.e. in } \Omega^{\delta} \text {, as } \varepsilon \rightarrow 0^{+} .
$$

Moreover, the uniform ellipticity condition $\mathrm{U}$ for $a=\left[a_{i j}\right]$ is preserved for $a^{(\varepsilon)}=\left[a_{i j}^{(\varepsilon)}\right]$. See, e.g., Subsection 5.3.1 and Appendix C.5 in E] for these and other properties of mollifiers.

We will rely on the existence of smooth solutions of the Dirichlet problem with smooth data. Namely, if $B_{R+\delta} \subset \Omega$, then there exists a unique solution $u_{\varepsilon} \in C^{\infty}\left(\overline{B_{R}}\right)$ of the problem

$$
L_{\varepsilon} u_{\varepsilon}:=\left(D, a^{(\varepsilon)} D u_{\varepsilon}\right)=0 \text { in } B_{R}, \quad u_{\varepsilon}=u^{(\varepsilon)} \text { on } \partial B_{R} .
$$

For our purposes, it suffices to have $u_{\varepsilon} \in C^{1}\left(\overline{B_{R}}\right)$.

Lemma 6.1. We have $u_{\varepsilon} \rightarrow u$ in $W^{1,2}(\Omega)$, i.e.,

$$
\int_{B_{R}}\left|u_{\varepsilon}-u\right|^{2} d x+\int_{B_{R}}\left|D u_{\varepsilon}-D u\right|^{2} d x \rightarrow 0 \text { as } \varepsilon \rightarrow 0^{+} \text {. }
$$

Proof. We use the integral identities corresponding to $L u=0$ and $L_{\varepsilon} u_{\varepsilon}=0$ in $B_{R}$, with a smooth function $\eta_{\varepsilon}:=u^{(\varepsilon)}-u_{\varepsilon}$ that vanishes on $\partial B_{R}$ :

$$
\int_{B_{R}}\left(a D u, D \eta_{\varepsilon}\right) d x=0 \text { and } \int_{B_{R}}\left(a^{(\varepsilon)} D u_{\varepsilon}, D \eta_{\varepsilon}\right) d x=0 .
$$

Then

$$
\nu \int_{B_{R}}\left|D \eta_{\varepsilon}\right|^{2} \leq \int_{B_{R}}\left(a^{(\varepsilon)} \eta_{\varepsilon}, D \eta_{\varepsilon}\right) d x=\int_{B_{R}}\left(v_{\varepsilon}, D \eta_{\varepsilon}\right) d x
$$

where $v_{\varepsilon}:=a^{(\varepsilon)}\left(D u^{(\varepsilon)}-a D u\right.$. We write

$$
\left|v_{\varepsilon}\right| \leq\left|a^{(\varepsilon)}\left(D u^{(\varepsilon)}-D u\right)\right|+\left|\left(a^{(\varepsilon)}-a\right) D u\right|=: F_{1, \varepsilon}+F_{2, \varepsilon} .
$$


By (6.2),

$$
F_{1, \varepsilon} \leq N \cdot\left|D u^{(\varepsilon)}-D u\right| \rightarrow 0 \text { in } L^{2},
$$

and also by the dominated convergence theorem we have $F_{2, \varepsilon} \rightarrow 0$ in $L^{2}$. By the CauchySchwarz inequality, from (6.5) it follows that $\left|D \eta_{\varepsilon}\right| \rightarrow 0$ in $L^{2}$. Using also the Poincaré inequality, we get $\eta_{\varepsilon} \rightarrow 0$ in $W^{1,2}$. Together with the convergence $u^{(\varepsilon)} \rightarrow u$ in $W^{1,2}$, we finally get (6.4).

Lemma 6.2. The functions $\left\{u_{\varepsilon}, 0<\varepsilon \leq \delta\right\}$ are uniformly bounded on $B_{R-\delta}$.

Proof. It is easy co check that $L_{\varepsilon}\left(u_{\varepsilon}^{2}\right) \geq 0$ in $B_{R}$. Indeed, for an arbitrary $0 \leq \eta \in$ $C_{0}^{\infty}\left(B_{R}\right)$ we have

$$
\int_{B_{R}}\left(a^{(\varepsilon)} D\left(u_{\varepsilon}^{2}\right), D \eta\right)=\int_{B_{R}}\left(a^{(\varepsilon)} D u_{\varepsilon}, 2 u_{\varepsilon} D \eta\right) .
$$

Since

$$
2 u_{\varepsilon} D \eta=D \eta_{0}-2 \eta D u_{\varepsilon}, \text { where } \eta_{0}:=2 \eta u_{\varepsilon} \in C_{0}^{\infty}\left(B_{R}\right),
$$

the previous expression is equal to

$$
-2 \int_{B_{R}} \eta\left(a^{(\varepsilon)} D u_{\varepsilon}, D u_{\varepsilon}\right) \leq 0
$$

This means that the $u_{\varepsilon}^{2}$ are a nonnegative subsolution uniformly bounded in $L^{1}\left(B_{R}\right)$. Now we can apply Theorem 3.5 with $u=u_{\varepsilon}^{2}, p=1$, and $\delta$ in place of $R$ :

$$
u_{\varepsilon}^{2}\left(x_{0}\right) \leq \frac{N}{\left|B_{\delta}\right|} \int_{B_{\delta}\left(x_{0}\right)} u_{\varepsilon}^{2} \leq N \int_{B_{R}} u_{\varepsilon}^{2} d x \leq N \quad \forall x_{0} \in B_{R-\delta} .
$$

Here $N$ denotes various different constants independent on $\varepsilon$. The lemma is proved.

Lemma 6.3. The functions $u_{\varepsilon}$ converge uniformly on $B_{R-2 \delta}$ as $\varepsilon \rightarrow 0^{+}$to a Hölder continuous function $u_{0}$ that coincides with $u$ a.e.

Proof. Indeed, estimate (5.4) for $u_{\varepsilon}$ in balls $B_{\rho}$ with different centers guarantees the compactness of $\left\{u_{\varepsilon}\right\}$ in $C\left(\overline{B_{R-2 \delta}}\right)$ and convergence to a function $u_{0}$. Since also $u_{\varepsilon} \rightarrow u$ in $L^{2}$, the limit function $u_{0}$ coincides with $u$ a.e., and $u_{0}$ satisfies (5.4).

On the grounds of Lemmas 6.1 6.3, the extension of results in $\$ 5$ from $u_{\varepsilon} \in C^{1}$ to $u \in W^{1,2}$ is straightforward. Note that we only used the convergence $u_{\varepsilon} \rightarrow u$ in $L^{2}$, though Lemma 6.1 provides convergence together with $D u_{\varepsilon} \rightarrow D u$ in $L^{2}$.

\section{REFERENCES}

[A63] A. D. Aleksandrov, Uniqueness conditions and bounds for the solution of the Dirichlet problem, Vestnik Leningrad. Univ. Ser. Mat. Meh. Astronom. 18 (1963), no. 3, 5-29; English transl., Amer. Math. Soc. Transl. (2) 68 (1968), 89-119. MR0164135

[DG57] E. De Giorgi, Sulla differenziabilità e l'analiticità delle estremali degli integrali multipli regolari, Mem. Accad. Sci. Torino. Cl. Sci. Fis. Mat. Nat. (3) 3 (1957), 25-43. MR 0093649

[E] L. C. Evans, Partial differential equations, 2nd ed., Grad. Stud. Math., vol. 19, Amer. Math. Soc., Providence, RI, 2010. MR2597943

[FS01] E. Ferretti and M. V. Safonov, Growth theorems and Harnack inequalities for second order parabolic equations, Contemp. Math., vol. 277, Amer. Math. Soc., Providence, RI, 2011, pp. 87112. MR:1840429

[HL] Q. Han and F. Lin, Elliptic partial differential equations, Courant Lecture Notes in Math., vol. 1, Amer. Math. Soc., Providence, RI, 1997. MR1669352

[GT] D. Gilbarg and N. S. Trudinger, Elliptic partial differential equations of second order, 2nd ed., Grundlehren Math. Wiss., Bd. 224, Springer-Verlag, Berlin, 1983. MR737190

[K85] N. V. Krylov, Second-order nonlinear elliptic and parabolic equations, Nauka, Moscow, 1985; English transl., Reidel, Dordrecht, 1987. MR815513 
[KS80] N. V. Krylov and M. V. Safonov, A property of the solutions of parabolic equations with measurable coefficients, Izv. Akad. Nauk SSSR. Mat. 44 (1980), no. 1, 161-175; English transl., Math. USSR-Izv. 16 (1981), no. 1, 151-164. MR563790

[La71] E. M. Landis, Second order equations of elliptic and parabolic type, Nauka, Moscow, 1971; English transl., Transl. Math. Monogr., vol. 171, Amer. Math. Soc., Providence, RI, 1998. MR1487894 (98k:35034)

[LSU] O. A. Ladyzhenskaya, V. A. Solonnikov, and N. N. Ural'tseva, Linear and quasilinear equations of parabolic type, Nauka, Moscow, 1967; English transl., Transl. Math. Monogr., vol. 25, Amer. Math. Soc., Providence, RI, 1968. MR0241821 (39:3159b)

[LU] O. A. Ladyzhenskaya and N. N. Ural'tseva, Linear and quasilinear elliptic equations, Nauka, Moscow, 1964; English transl., Acad. Press, New York, 1968. MR0244627

[M60] J. Moser, A new proof of de Giorgi's theorem concerning the regularity problem for elliptic differential equations, Comm. Pure Appl. Math. 13 (1960), 457-468. MR0170091

[M61] , On Harnack's inequality for elliptic differential equations, Comm. Pure Appl. Math. 14 (1961), 577-591. MR0159138 (28:2356)

[M64] J. Moser, A Harnack inequality for parabolic differential equations, Comm. Pure Appl. Math. 17 (1964), 101-134; and correction in: Comm. Pure Appl. Math. 20 (1967), 231-236. MR0159139 (28:2357) MR0203268 (34:3121)

[S80] M. V. Safonov, Harnak's inequality for elliptic equations and Hölder property of their solutions, Zap. Nauchn. Sem. Leningrad. Otdel. Mat. Inst. Steklov. (LOMI) 96 (1980), 272-287; English transl., J. Soviet Math. 21 (1983), no. 5, 851-863. MR.579490

School of Mathematics, University of Minnesota

E-mail address: safonov@math.umn.edu

Received 19/FEB/2015

Originally published in English 